\title{
Mathematical models of p53-microRNA and their applications
}

\author{
S.D. Senotrusova ${ }^{1,2 *}$, O.F. Voropaeva ${ }^{1}$ \\ ${ }^{1}$ Institute of Computational Technologies SB RAS, Novosibirsk, Russia \\ ${ }^{2}$ Novosibirsk State University, Novosibirsk, Russia \\ *e-mail: senotrusova.s@mail.ru
}

Key words: tumor marker, p53, miRNA, delay differential equations, numerical simulation

Motivation and Aim: One of the priorities of modern biomedical research is the search for effective biomarkers for early cancer detection and other serious diseases related with dysfunction of processes of cell death. The p53 protein (tumor necrosis factor), involved in many life and death processes, including the formation of tumors and aging, is expressed in all the cells of the organism. Mdm2 protein is considered to be the key negative $\mathrm{p} 53$ regulator [1]. It is known that $\mathrm{p} 53$ regulates the class of microRNAs (miRNA), which are characterized as the most important intermediates of p53 in tumor control. Thus, the investigation of the function of the p53 protein and updating of the diagnostic properties of miRNA is paramount both for developing new approaches to cancer treatment and determining the prevention strategy for many diseases, including measures to slow the aging processes.

Methods and Algorithms: We propose in this work a hierarchy of mathematical models of the dynamics of network p53-Mdm2-microRNA for miRNA class with a direct positive connection with $\mathrm{p} 53$. These models include the systems of three nonlinear equations with delaying arguments.

Results: All obtained numerical solutions have a sufficiently clear biomedical meaning. A comparative analysis of models in a wide range of parameters is performed. Stress situations associated with the emergence of an imbalance in the rates of p53 and Mdm2 generation and degradation and also with disturbances in the mechanism of in implementation interaction of proteins regulated within the considered models through the constants of dissociation have been studied. Numerical investigation of the microRNAs functioning in conditions of the deregulation of p53 and p53-Mdm2-network is carried out. The deregulation of microRNA in detail is studied. The situations in which p53, its inhibitor Mdm2 and microRNAs exhibit critical properties for the patient's status and can be identified as diagnostic markers of cancer and neurodegenerative disease are studied. The results of numerical analysis are in good agreement with the data of clinical and laboratory studies of known microRNAs.

Conclusion: According to the results of these investigations, p53-responsive microRNA of given class can be used to clarify function of p53 as a biomarker of cancer and neurodegenerative diseases [1]. If the network p53-microRNA functions normally, then microRNA duplicates diagnostic properties of $\mathrm{p} 53$. The variants of microRNAs deregulation are found, where microRNAs are an even better marker for disease than p53. The most significant differences in the analysis results are due not to the introduction of additional microRNA feedback with Mdm2, as could be expected, but to the refinement of the approximation of the interaction function for the p53-miRNA. We present other examples of the application of the developed models.

\section{References}

1. Voropaeva O.F., Senotrusova S.D., Shokin Yu.I. (2017) Numerical study of diagnostic properties of p53dependent microRNA. Russ. J. Numer. Anal. Math. Modelling. 32(3):203-213. 